LBL-32979

\title{
REDUCED MASS PERSISTENT SWITCHES FOR LARGE SUPERCONDUCTING MAGNETS IN SPACE
}

LBL -32979

M. A. Green

DE93 004782

Lawrence Berkeley Laboratory

University of California

Berkeley, CA 94720

\author{
1992 Applied Superconductivity Conference \\ Hyatt Regency Hotel \\ Chicago IL, USA \\ August 24-28, 1992 \\ To be published in \\ IEEE Transactions on Applied Superconductivity 3
}

\footnotetext{
*This work was performed at the Lawrence Berkeley Laboratory with the support of the Ofiice of Astrophysics, NASA and the Director of the Office of Energy Research, Office of High Energy and Nuclear Physics, High Energy Physics Division, U. S. Department of Energy under Contract No. DE-AC03-76SF00098.
} 


\title{
REDUCED MASS PERSISTENT SWITCHES FOR LARGE SUPERCONDUCTING MAGNETS IN SPACE
}

\author{
Michael A. Green \\ Lawrence Berkeley Laboratory \\ Berkeley CA, 94720, USA
}

\begin{abstract}
Superconducting magnets in space must operate in the persistent mode. This paper describes the characteristics of low mass niobium titanium persistent switches for low mass superconducting magnets which are designed to quench protect themselves through the quench back process. (ihe whole coil is driven normal shortly after the quench has started and the magnet stored energy is taken up by the coil and the persistent switch.) The concept of using a resistor and diode in parallel with the persistent switch to reduce the overall mass of the persistent switch system and the helium consumption during magnet charging is discussed in the report. A 1.4 meter ciameter free-flyer version of the $11.6 \mathrm{MJ}$ stored energy ASTROMAG magnet and its persistent switch is presented as an example [1].
\end{abstract}

\section{SELECTION OF MATERIALS FOR THE PERSISTENT SWITCH}

The use of a high RRR material is desirable from the standpoint of stability of the superconductor in the switch. On the other hand, a low RRR material is desirable from the standpoint of switch and magnet safety should the switch turn normal while the magnet is fully charged in the persistent mode. A high energy per unit mass from the operating temperature to $300 \mathrm{~K}$ permits one to discharge more of the magnet's energy through the switch. For example, the ASTROMAG magnet, shown in Fig. 1, has a stored energy of $11.6 \mathrm{MJ}$ when operating at its design current. If the entire energy of the magnet were discharged through the persistent switch, one might make the switch from $68 \mathrm{~kg}$ of aluminum. If the switch were made from a copper alloy, its mass would be over $140 \mathrm{~kg}$ for the same discharge condition. Because quench back is used for quench protection in magnets for space such as the ASTROMAG magnet, less material is needed in the persistent switch. Under these circumstances, the average resistance of the persistent switch during a coil discharge determines the total energy entering the switch when it turns normal.

It turns out that high RRR purc inetals are not good material choices for the stabilizer for switches. The low RRR copper nickel alloys, which are used commonly in persistent switches [2,3], appear also to be good materials for a space magnet switch because they have an RRR very nearly one. The aluminum alloys will absorb more heat per unit mass, but RRR is substantially above one. As a result, aluminum stabilizers will perform no better than the low RRR copper alloy stabilizers which can easily be codrawn with the niobium titanium in the switch.

R.A.anuscript received August 24, 1992

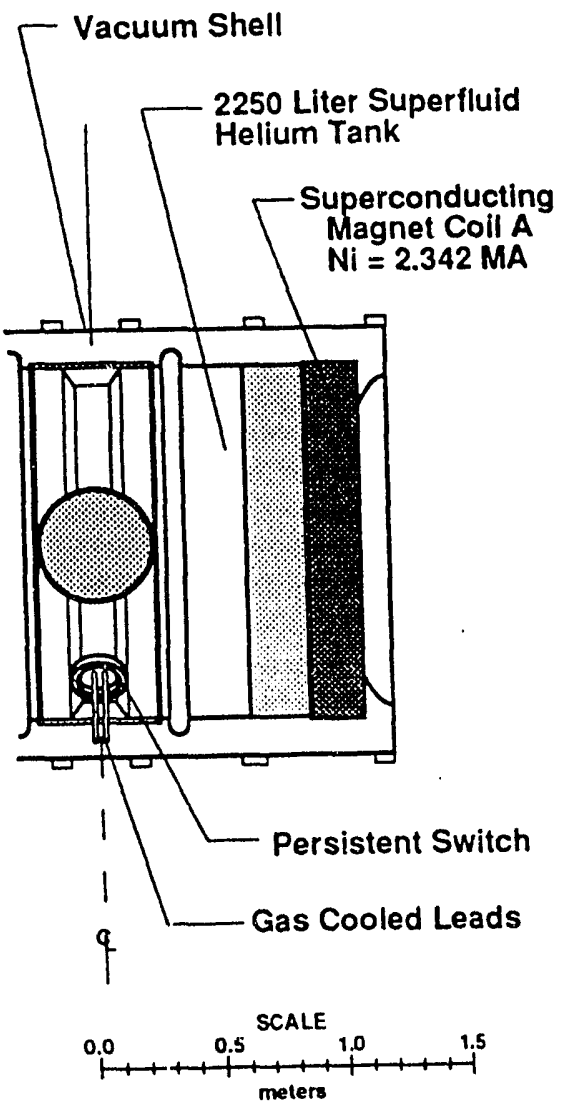

Fig. 1 The ASTROMAG Magnet Showing the Location of the Main C'oils and the Persistent Switch

\section{THE PERSISTENT SWITCH RESISTANCE}

The resistance of the persistent switch when it is closed is dictated by the mirimum allowable decay time constant for the current in the magnet whic? it is in the charged and persistent state. The maximum rate of stored energy decay allowed for ASTROMAG is one percent per year. This translates to a magnet circuit decay time constant of $6.31 \times$ $10^{9}$ seconds. The ASTROMAG magnet circuit has a self inductance of $36.14 \mathrm{H}$. Therefore, the maximum allowable resistance of the magnet circuit is 5.73 nano-ohms. Ahout one third of this resistance (about 2 nano-ohms) can be allocated to the persistent switch. The switch itself can be made from a single piece of super-conductor. The joints which connect the switch to the magnet coils should have a resistance of less than 1 nano-ohm. With proper care, these joints can be made in a low field region to insure a low joint resistance.

The open circuit resistance of the switch is driven by several factors. Factors which favor the maximization of the 
switch (jen circuit resistance include: 1) a reduction of the heat generated in the switch during charging or discharging, 2) a reduction of the time needed to charge the coil (this is related to the previous factor), and 3 ) a reduction of the switch switching time. Factors which favor a reduction of the open circuit resistance are: 1) the minimization of the voltage generated across the switcil and the magnet coils should the switch open (turn normal) at full current and 2) the fact that the mass of the switch is proportional to its open circuit resistance (this will be shown later).

Heat generated in the persistent switch during charging and discharging is proportional to the charge voltage squared and inversely proportional to the switch open circuit resistance $R_{s}$. If the magnet is charged at a uniform rate, the energy put into the superfluid helium $E_{H}$ from the switch, the coil quench back circuit, and heat flow down the leads, can be estimated using the following expression:

$$
E_{H}=0.002 i_{0} L_{c}+\frac{L_{1}^{2} i_{o}^{2}}{t_{c} R_{S}}+\frac{2 M_{12} i_{0}^{2}}{t_{c} R_{2}}
$$

where $i_{O}$ is the operating current of the magnet; $l_{c}$ is the magnet charge time; $L_{1}$ is the magnet circuit self inductance; $R_{s}$ is the open circuit resistance of the switch; $M_{12}$ is the mutual inductance between the magnet circuit and the quench back circuit in each coil; and $R_{2}$ is the coil quench back circuit resistance. From Eq. 1, it is clear that there is a magnet sharge time $t_{c}$ which results in a minimum amount of energy $E_{H}$ being deposited into the superfluid helium tank. If one differentiates Eq. I with respect to $t_{c}$, the following expression for the optimum value of the charge time $t_{\text {co }}$ will result:

$$
\mathcal{L}_{\mathrm{O}}=22.36 \mathrm{i}_{\mathrm{o}} 0.5\left[\frac{\mathrm{L}_{1}^{2}}{\mathrm{R}_{\mathrm{S}}}+\frac{2 \mathrm{M}_{12}{ }^{2}}{\mathrm{R}_{2}}\right]
$$

The higher the switch normal circuit resistance $R_{s}$, the smaller the energy $E_{H}$ and the shorter the optimum value of the charge time $t_{\text {co }}$ are. When $L_{1}=36.1 \mathrm{H}, \mathrm{R}_{\mathrm{s}}=1.2 \Omega$ $M_{12}=1.29 \mathrm{H}, R_{2}=0.008 \Omega$ and $i_{0}=800 \mathrm{~A}$, the value of $\mathrm{t}_{\mathrm{co}}$ would be $24510 \mathrm{~s}(6.81 \mathrm{hr})$ and $\mathrm{E}_{\mathrm{H}}$ would be $78440 \mathrm{~J}$.

It is desirable, from the standpoint of optimum charge time and the energy deposited into the superfluid helium tank, to increase the open circuit resistance of the switch. The two arguments against having a high switch open circuit resistance is the voltage generated when the switch turns normal and the mass of the switch. For an $800 \mathrm{~A}$ magnet, the switch open circuit resistance should not exceed 1.0 to $1.5 \mathrm{ohms}$ unless the persistent switch is in parallel with a diode shunt resistor set.

\section{THE PERSISTENT SWITCH MASS}

The mass of the persistent switch for a space magnet is directly related to the resistance of the switch (or parallel resistor) and the decay time for the current in the magnet once the persistent switch has gone normal. If the decay time constant of the magnet is very long so that virtually all of the magnet stored energy is deposited into the persistent switch, one chooses a switch (or parallel resistor) material such as aluminum. In this case, the maximum mass for the switch for an $11.6 \mathrm{MJ}$ magnet is $68 \mathrm{~kg}$. Since the magnet is caused to quench back by the switch turning normal (a requirement), most of the magnet energy will be dissipated in the magnet coils rather than the persistent switch. One can estimate an upper limit for the switch mass if one knows the decay of the magnet coil through itself after quench back has occurred. The final temperature in a persistent switch with RRR $=1$ is directly related to the current decay in the magnet circuit through the following expression;

$$
\int_{0}^{\infty} j(t)^{2} d t=\frac{r}{(r+1)} \frac{d \Delta H}{\rho}
$$

where $j(t)$ is the current density in the switch material as a function of time $\mathrm{t}$; $\Delta \mathrm{H}$ is the material enthalpy change per unit mass from 2 to $300 \mathrm{~K}$; $d$ is the switch material mass density; and $\rho$ is the room temperature resistivity of the switch material. The mass of the persistent switch can be approximated using the following expression;

$$
\operatorname{mass}=\left[\mathrm{i}_{0}{ }^{2} \frac{\mathrm{R}_{\mathrm{S}}}{\Delta H}\right] \int_{0}^{\infty} \zeta_{(t)^{2} d t}
$$

where $\zeta(t)=i(t) / i_{0}$ and $i_{0}$ is the current at the start of the magnet discharge. From Eq. 4, it is clear that the switch mass is proportional to its resistance, propcrtional to the magnet current squared, and inversely proportional to the energy per unit mass needed to change the temperature from 2 to $300 \mathrm{~K}$. The value of $R_{S}$, which should be used in Eq. 4 , is the switch resistance at temperature $300 \mathrm{~K}$. If the correct switch material is used, the value of $R_{S}$ will be only a few percent higher than the switch resistance at $10 \mathrm{~K}$. The total integral of $S_{(t)}$ squared with time from the moment the switch turns normal to infinity (when the current in the coils and the switch is zero) will be about 1.8 for the ASTROMAG magnet. If one uses a value of $R_{s}=1.2 \Omega$, $\Delta \mathrm{H}=80000 \mathrm{~J} \mathrm{~kg}^{-1}$, and the magnet current $\mathrm{i}_{0}=800 \mathrm{~A}$, the active mass for the switch will be $17.3 \mathrm{~kg}$. The only way to reduce the switch mass is to put it in parallel with a resistor and diode set. The overall resistance of the switch, resistor plus diode set (once current flows in the diodes), 
need only be the resistance needed to solidly induce quench back in the magnet coils.

\section{MINIMUM SWITCH SYSTEM RESISTANCE NEEDED TO INDUCE QUENCH BACK IN THE MAGNET}

The ASTROMAG magnet coils will be protected during a quench using the quench back phenomena [4]. The coil for ASTROMAG will have two layers of pure $R R R=1000$ aluminum which forms a shorted secondary winding with a resistance of $0.008 \Omega$ at $20 \mathrm{~K}$ or less. This circuit is inductively coupled to the main magnet coil. The mutual inductance between the quench back circuit and the main ASTROMAG coils is about $1.29 \mathrm{H}$. The coupling coefficient is about 0.96 . When the main coil current decays, due to a normal region forming in the coil or the persistent switch turning normal, a current is induced in the secondary circuit. The quench back circuit current heats up the quench back circuit and nearby coil superconductor causing the whole coil to go normal all at once. The allowable time for quench back is determined by the allowable hot spot temperature (usually $300 \mathrm{~K}$ ) for the magnet, the material in the superconductor and the quench back circuit, and the matrix current density in the superconducting windings.

The value of the minimum switch resistance needed to induce quench back $R_{\min }$ can be estimated with the following expression;

$$
\mathrm{R}_{\min }=\left[\frac{\mathrm{L}_{1}}{\tau_{2}} \frac{\mathrm{N}_{2}}{\mathrm{~N}_{1}} \frac{\mathrm{A}_{\mathrm{C} 2}}{\mathrm{i}_{\mathrm{o}}}\right]\left[\frac{\Delta \mathrm{H}_{2}}{\rho_{2} \mathrm{t}_{\mathrm{QR}}}\right]^{0.5}
$$

where $\tau_{2}$ is the $\mathrm{L}$ over $\mathrm{R}$ time constant for the quench back circuit (about 12 seconds); $\mathrm{N}_{2}$ is the number of turns in the quench back circuit ( $N_{2}=209$ turns); $N_{1}$ is the number of turns in the coil well coupled with the quench back circuit ( $N_{1}=2928$ turns); $A_{c 2}$ is the cross-sectional area of the quench back circuit conductor $\left(A_{c 2}=3 \times 10^{-6} \mathrm{~m}^{2}\right) ; \Delta H_{2}$ is the enthalpy per unit volume to raise the quench back circuit material to $10 \mathrm{~K}\left(\Delta \mathrm{H}_{2}=13200 \mathrm{~J} \mathrm{~m}^{-3}\right)$; and $\rho_{2}$ is the low temperature resistivity of the quench back circuit material ( $\left.\rho_{2}=2.5 \times 10^{-11} \mathrm{ohm} \mathrm{m}\right) . L_{1}(36.2 \mathrm{H}), i_{o}(800 \mathrm{~A})$, and $\mathrm{t}_{\mathrm{QR}}$ is the required back time for a safe quench $(0.2 \mathrm{sec})$. From Eq. 5, the value of $R_{\min }=0.046$ ohms. The prediction of quench back times has been fairly accurate for thin solenoids [5], but for thicker coils, such as the ASTROMAG coils, such predictions may not be as accurate. As a result, one should use a minimum value of the switch circuit resistance several times the calculated value. A minimum switch resistance of $0.3 \mathrm{ohms}$ has been chosen for the ASTROMAG persistent switch circuit.

\section{THE PERSISTENT SWITCH WITH A PARALLEL RESISTOR AND COLD DIODES}

Silicon diodes require a finite voltage to drive current through them in the forward direction. If the voltage is smaller than the voltage needed to drive the current through the diode in the forward direction, no current will be carried. At $2 \mathrm{~K}$, this voltage is around 2.5 volts. A circuit diagram of the magnet with a persistent switch in parallel with diodes and a protection resistor is shown in Fig. 2. The advantages of the circuit with the diodes and parallel resistor are as follows: 1) The persistent switch open circuit resistance can be much higher. This means that less heat is dissipated in the switch during charging. 2) The optimum charging time is shorter for the switch with the resistor in parallel. Less superfluid helium is needed to charge or discharge the magnet. 3) The discharge voltage of the magnet through the switch is lower for the case with the parallel resistor. 4) The overall mass of the persistent switch system is smaller for the persistent switch with diodes and the parallel resistor because the element which absorbs the energy from the coil is now the parallel resistor instcad of the switch.

The only disadvantage of the resistor and the diodes in parallel with the switch is the additioral complexity of having the diodes and the reliability associated with increased system complexity. Cold diodes in themselves have proved to be quite reliable. Figure 3 shows a schematic crosssection view of a $4.64 \mathrm{ohm}$ bifilar persistent switch in parallel with a pair of opposing cold diodes and a $0.3 \mathrm{ohm}$ bifilar wound resistor (See Fig. 2.). The switch is assumed to have a copper nickel matrix and the resistor is assumed to be made from the same copper nickel alloy. The mass of the persistent switch system shown in Fig. 3 is about 8.9 $\mathrm{kg}$ as compared to a switch mass of $18.5 \mathrm{~kg}$ (17.3 kg active switch mass) without a parallel resistor.

\section{CONCLUSIONS}

The main coil ASTROMAG persistent switch can be made so that it will fit in a $350 \mathrm{~mm}$ diameter space on the surface of the superfluid helium tank at a place halfway between the ASTROMAG main coils. The persistent switch can be combined with the magnet gas cooled electrical leads. The persistent switch system mass can be as low as $8.9 \mathrm{~kg}$, when the switch is combined with a $\mathrm{Cu}$ $\mathrm{Ni}$ resistor and a pair of cold diodes. The ASTROMAG persistent switch system can be made so that the switch can be turned normal while it carries the full current of the magnet. When the switch turns normal, the entire magnet will turn normal through quench back. When the switch is in parallel with a $0.3 \mathrm{ohm}$ resistor, the switch resistance can be greater than 5 ohms which has the effect of allowing one to charge the magnet in times as short as 4 hours. Under $2.5 \mathrm{~kg}$ of helium is required to charge or discharge the ASTROMAG magnet and cool the gas cooled electrical leads when the switch is in parallel with a resistor and cold diodes. 


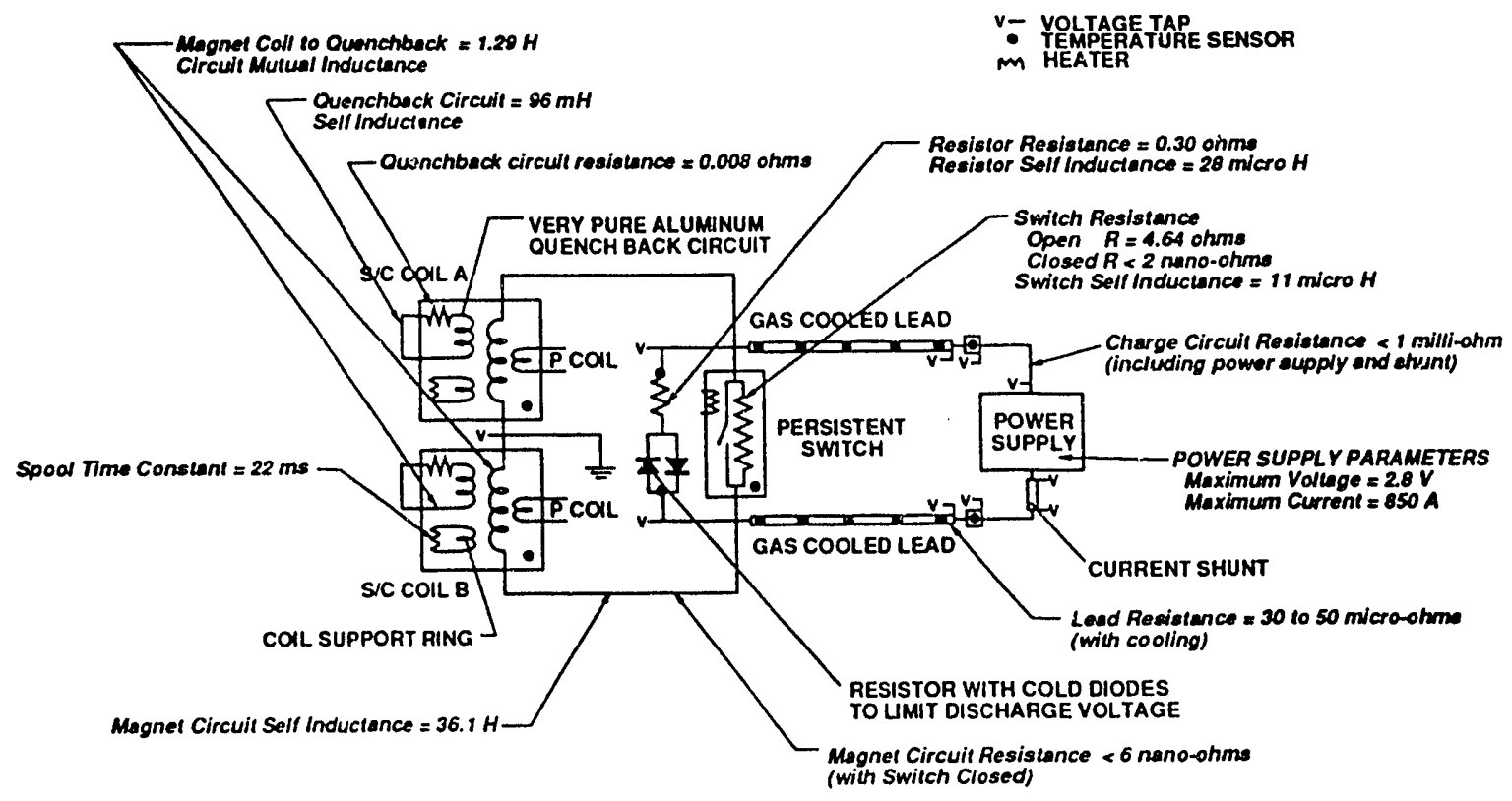

Fig. 2. A schematic Diagram of the ASTROMAG Solenoid and Persistent Switch with a Parallel Resistor and Diodes

\section{ACKNOWLEDGEMENT}

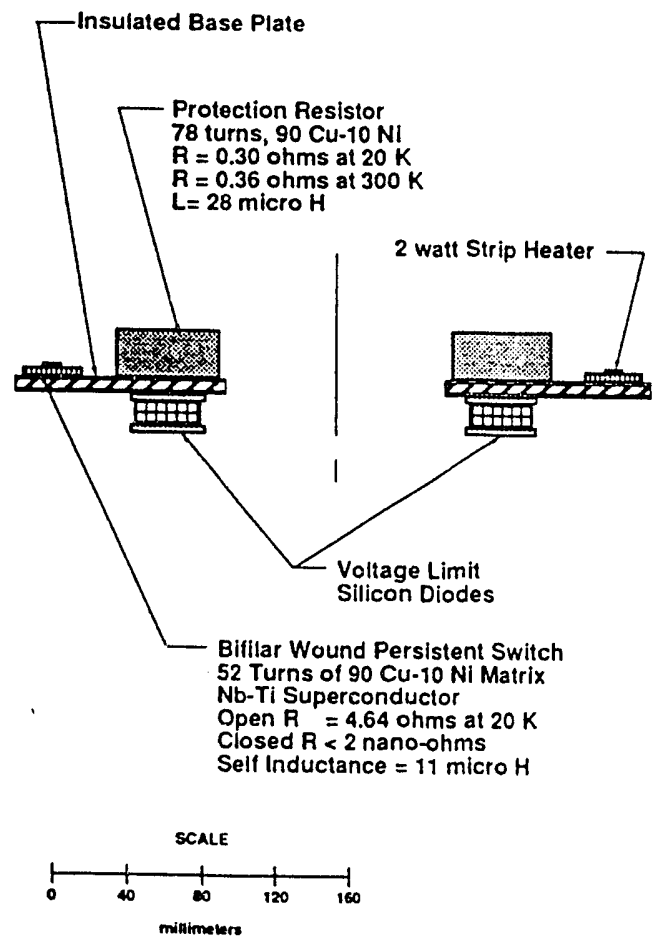

This work was supported by the office of Astrophysics of NASA and the Office of Basic Energy Science, United States Department of Energy under the Lawrence Berkeley Laboratory contract number DE-AC03-76SF00098.

\section{REFERENCES}

[1] M. A. Green and G. F. Smoot, "The ASTROMAG Superconducting Magnet Facility Configured for a Free Flying Satellite," Cryogenics 32, No. 2, p 91, (1992)

[2] M. N. Wilson, Superconducting Magnets, Section 11.2, Persistent Switches, pp 272-275, Oxford Claredon Press, Oxford, UK (1983).

[3] M. A. Green, "A Design for a Reduced Mass Persistent Switch for the Free Fiyer Version of ASTROMAG", Lawrence Berkeley Laboratory Report LBID-1787, Sept. 1991

[4] M. A. Green, "The Role of Quench Back in Quench Protection of a Superconducting Solenoid," Cryogenics 24, p 659, LBL-16547, Dec. 1984.

[5] M. A. Green, "PEP-4, TPC Superconducting Magnet, A Comparison of Measured Quench Back Time with Theoretical Calculations of Quench Back Time in Four Thin Superconducting Magnets," Lawrence Berkeley Laboratory Report LBID-771, August 1983. 

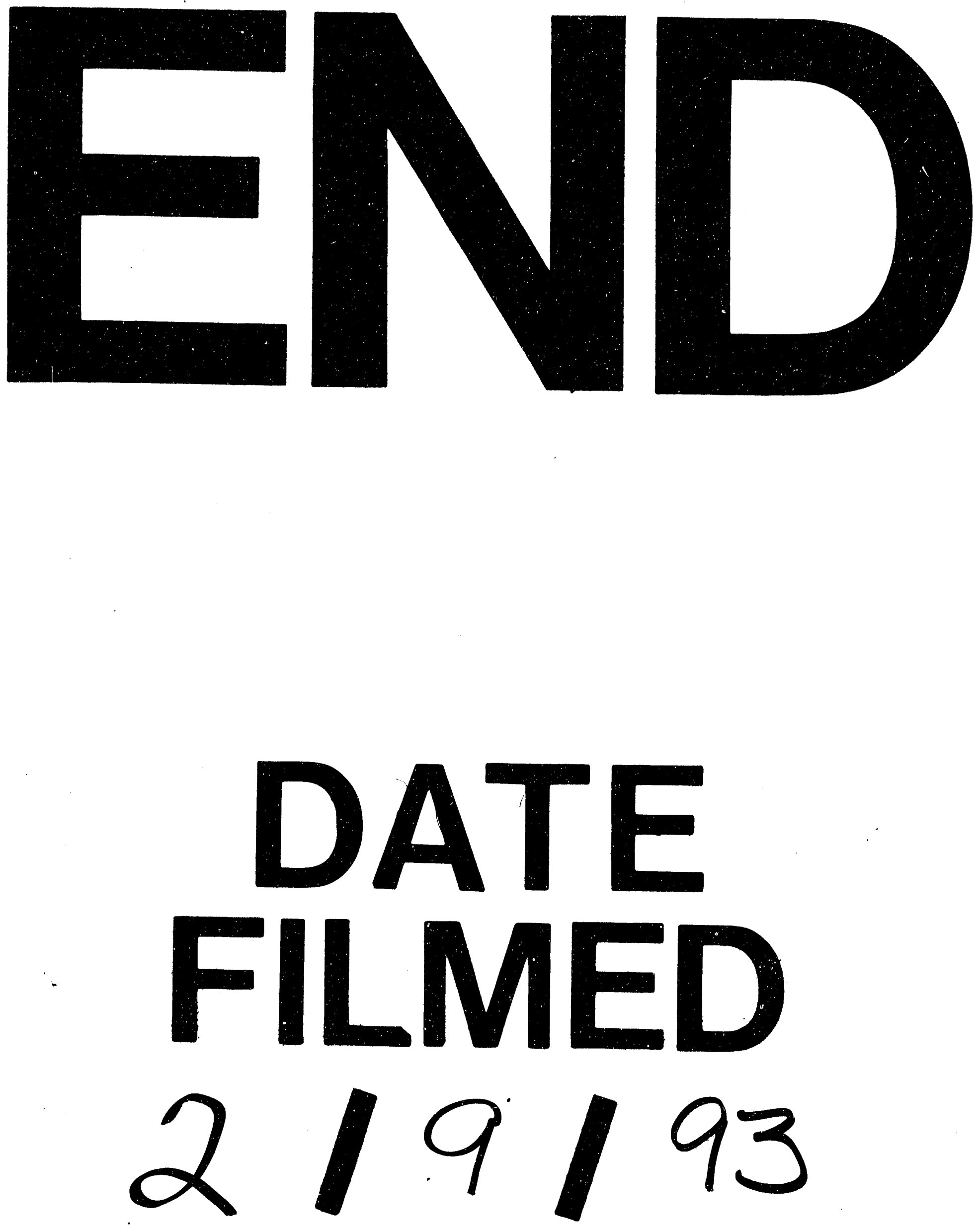
